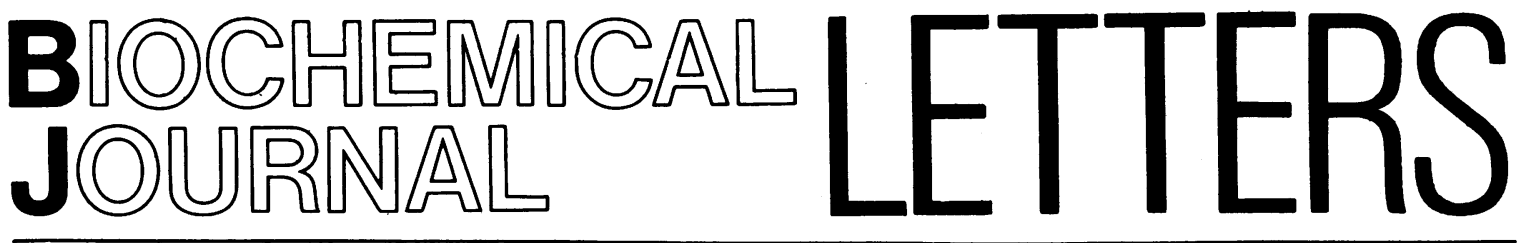

\section{'Chymopapain $\mathbf{S}$ ' is chymopapain $\mathbf{A}$}

Over the last few years we have isolated and characterized the constituent cysteine proteinases of the dried latex of Carica papaya obtained from a number of sources. The general picture (see Baines \& Brocklehurst, 1982a; Brocklehurst \& Salih, 1983) appears to be that the latex contains two monothiol cysteine proteinases, namely papain, EC 3.4.22.2, (for which we proposed the auxilliary name, papaya peptidase I) and papaya peptidase II (originally called papaya peptidase A by Schack, 1967). The other cysteine proteinases present are dithiol cysteine proteinases, and we suggested that the general name 'chymopapain' should be given to this group of enzymes. All of these chymopapains contain one thiol group per molecule that is essential for catalytic activity, and one nonessential thiol group. The uses of disulphides containing the 2-pyridyl moiety in the isolation of thiol enzymes by covalent chromatography and in the study of thiol group characteristics have been reviewed (Brocklehurst, 1982). By using the commercially available reagent, $2,2^{\prime}$-dipyridyl disulphide, as a thiol-specific two-protonic-state reactivity probe, it is particularly easy to distinguish chymopapain A from the various chymopapains B $\left(B_{1}, B_{2}\right.$ and $B_{3}$, which appear to be rather similar to each other in some respects) by the form of the $\mathrm{pH}$-dependence of the second-order rate constant, $k$, for the reactions of this reagent with the essential thiol groups of these enzymes (Brocklehurst et al., 1980; Baines \& Brocklehurst, 1982a; Brocklehurst \& Salih, 1983).

The general picture of the range and nature of the variation in the cysteine proteinases commonly present in commercially available papaya latex (see Brocklehurst \& Salih, 1983) was made uncertain by the interesting report (Khan \& Polgár, 1983) that papaya latex supplied by Sigma does not contain either chymopapain A or any chymopapain $B$ but instead contains an enzyme with 'hybrid' kinetic properties designated by Khan \& Polgár (1983) as 'chymopapain S'.

We now report that the papaya latex currently supplied by Sigma $(a)$ when freed from papain and papaya lysozyme by $\left(\mathrm{NH}_{4}\right)_{2} \mathrm{SO}_{4}$ precipitation and subjected to ion-exchange chromatography provides an elution pattern of its protein constituents closely similar to that reported by Khan \& Polgár
(1983) and (b) does contain chymopapain A and the chymopapains $B$ as well as papain and papaya peptidase II. The constituent of the latex that corresponds to chymopapain $\mathrm{S}$ both in its position in the elution profile and in its kinetic properties is indistinguishable from chymopapain A (prepared as described by Baines \& Brocklehurst, 1982a) in its reactivity characteristics towards $2,2^{\prime}$-dipyridyl disulphide and in its activity loss behaviour consequent on thiol modification. These are the two criteria on which Khan \& Polgár (1983) concluded that 'chymopapain S' had the properties of chymopapain $\mathbf{A}$ in acidic media but those of chymopapain B in alkaline media. The reactivity characteristics of the essential thiol group of chymopapain A towards 2,2'-dipyridyl disulphide are strikingly different from those of any other cysteine proteinase studied to date (Brocklehurst $\boldsymbol{e t}$ al., 1980, and see, e.g., Baines \& Brocklehurst, $1982 b$ ). Thus $k$ is $\mathrm{pH}$-independent between $\mathrm{pH} 3$ and $\mathrm{pH} 5.5$ and is approx. $2.5 \times 10^{3} \mathrm{M}^{-1} \cdot \mathrm{s}^{-1}$. For other cysteine proteinases the $\mathrm{pH}-k$ profile in the $\mathrm{pH}$ region 2-6 is bell-shaped, the maximal value of $k$ being at least $1 \times 10^{4} \mathrm{M}^{-1} \mathrm{~s}^{-1}$. In this respect 'chymopapain $\mathbf{S}$ ' and chymopapain $\mathbf{A}$ are indistinguishable, as was concluded by Khan \& Polgár (1983).

The conclusion of Khan \& Polgár (1983) that in alkaline media 'chymopapain S' possessed characteristics of chymopapain B rather than those of chymopapain A was arrived at by comparison of their Tsou Chen-Lu (1962) plot for the 'chymopapain $S^{\prime} / 2,2$ '-dipyridyl disulphide system obtained at pH9 with those reported previously (Baines \& Brocklehurst 1982a) for the chymopapain A/2,2'dipyridyl disulphide system at pH8.6 and for the chymopapain B (now known to be chymopapain $\left.\mathrm{B}_{3}\right) / 2,2^{\prime}$-dipyridyl disulphide system at $\mathrm{pH}$ 8.9. The method involves allowing an enzyme to react with a modifying reagent specific for a particular type of functional group whose reaction causes loss of catalytic activity. The number of essential groups per molecule is determined by measuring the residual catalytic activity $(a)$ and the number of groups modified in samples of partially modified enzyme. When the groups have similar reactivities, the number of essential groups per molecule $(i)$ is found from plots of $a^{1 / i}(i=1,2$ etc.) versus the number of groups modified per molecule, where only the correct value of $i$ provides a linear plot. 
When the essential and non-essential groups have sufficiently different reactivities, the form of the plots can reveal which group is essential for activity. In our previous paper (Baines \& Brocklehurst, 1982a) the shapes of the Tsou Chen-Lu plots for the chymopapain $A / 2,2^{\prime}$-dipyridyl disulphide and chymopapain $B\left(B_{3}\right) / 2,2$ '-dipyridyl disulphide systems at $\mathrm{pH}$ values 8.6 and 8.9 respectively were shown to be quite different. In the former, $a$ decreases linearly to zero as the total number of thiol groups becomes modified, whereas the latter is markedly biphasic, $a$ decreasing only gradually until approx. $50 \%$ of the total number of thiol groups has been modified and then decreasing in a steeper linear manner, to reach zero when $100 \%$ of the total thiol has been modified. It should be noted that the legend to Fig. 3 of Baines \& Brocklehurst (1982a), in which the Tsou Chen-Lu plots are shown and described, correctly identifies the various plots, Figs. 3(a)-3(e). Unfortunately the letters assigned to these Figures in the text on pages 135 and 137 are printed incorrectly, although these errors are readily discerned as such from the description of the various plots in these passages of text, and the situation is quite clear when the text is read in conjunction with Fig. 3.

The Tsou Chen-Lu plot reported by Khan \& Polgár (1983), when taken together with the published data on chymopapains $\mathbf{A}$ and $\mathbf{B}$, does lead logically to their deduction of the existence of the new enzyme chymopapain S. It is important now, however, to report the new information that the form of the Tsou Chen-Lu plot for the chymopapain A/2,2'-dipyridyl disulphide system changes markedly with $\mathrm{pH}$ in alkaline media. Although it is distinct from that for the chymopapain $\mathrm{B}_{3} / 2,2^{\prime}$-dipyridyl disulphide system at $\mathrm{pH} 8.6$ as was reported previously (Baines \& Brocklehurst, $1982 a$ ), it is similar to that for the chymopapain $\mathrm{B}_{3} / 2,2$-dipyridyl disulphide system at $\mathrm{pH} 9$ and above. The pH-dependence of the form of the Tsou Chen-Lu plot derives from the forms of the $\mathrm{pH}-k$ profiles for the reactions of 2,2'-dipyridyl disulphide with the non-essential and essential thiol groups of chymopapain A. In both profiles (B. S. Baines, E. Salih \& K. Brocklehurst, unpublished work), $k$ increases with $\mathrm{pH}$ in alkaline media; below pH8.6 the reactivity of the essential thiol group is greater than that of the non-essential thiol group; the profiles cross at approx. $\mathrm{pH} 8.6$ and at higher $\mathrm{pH}$ yalues the reactivity of the non-essential thiol group becomes approx. 2-3 times greater than that of the essential thiol group. This difference in reactivity is sufficient to provide a Tsou Chen-Lu plot for the chymopapain A/2,2'-dipyridyl disulphide system at $\mathrm{pH} 9$ similar to that characteristic of the chymopapain $B_{3} / 2,2^{\prime}$-dipyridyl disulphide system in alkaline media. The $\mathrm{pH}-k$ profiles for the analogous reactions of chymopapain $B_{3}$ cross at approx. pH6 and the reactivity of the nonessential thiol group of this enzyme in alkaline media is always considerably greater than that of the essential thiol group, which provides for the biphasic nature of the Tsou Chen-Lu plot for this system in alkaline media.

We have shown that both chymopapain $A$ prepared from the spray-dried latex from Zaire and the second-least basic cysteine proteinase in Sigma papaya latex (i.e. the least basic cysteine proteinase isolated by ion-exchange chromatography after precipitation of papain, which is itself the least basic proteinase) are characterized by the same type of $\mathrm{pH}$-dependent thiol reactivity towards 2,2'-dipyridyl disulphide in acidic media and by the same type of pH-dependent Tsou ChenLu plots in alkaline media (i.e. linear at $\mathrm{pH} 8.6$ and biphasic at pH9 and above).

The inescapable conclusion is that "chymopapain $S^{\prime}$ is chymopapain $A$.

We thank the Science and Engineering Research Council for support, including a Research Studentship for E. S. and a CASE Award (with Powell and Scholefield Ltd.) and a Postdoctoral Research Assistantship for B. S. B.

Keith BROCKLEHURST, Baldev S. BAINES,* Erdjan SALIH and Constantina HATZOULIS Department of Biochemistry, Medical College of St. Bartholomew's Hospital, University of London, Charterhouse Square, London ECIM 6BQ, U.K.

(Received 13 January 1984)

Baines, B. S. \& Brocklehurst, K. (1982a) J. Protein Chem. 1, 120-139

Baines, B. S. \& Brocklehurst, K. (1982b) Biochem. J. 205, 205-211

Brocklehurst, K. (1982) Methods Enzymol. 87C, 427-469

Brocklehurst, K. \& Salih, E. (1983) Biochem. J. 213, 559560

Brocklehurst, K., Baines, B. S. \& Mushiri, M. S. (1980) Biochem. J. 189, 189-192

Khan, I. U. \& Polgár, L. (1983) Biochim. Biophys. Acta 760, 350-356

Schack, P. (1967) C. R. Trav. Lab. Carlsberg 36, 67-83

Tsou Chen-Lu (1962) Sci. Sin. 11, 1535-1558

\footnotetext{
* Present address: Department of Microbiology, Queen Elizabeth College, London, U.K.
} 